\title{
Detection of Mismatch Repair and Microsatellite Instability in Colorectal Cancer Patients
}

\author{
Muhammad Ishaque Faizee \\ International Islamic University of Malaysia
}

Presenter: Muhammad Ishaque Faizee

Introduction: Colorectal cancer is the third most common cancer worldwide. Microsatellite instability (MSI) contributes to be one of the main mechanisms in colorectal cancer. Individuals with MSI tumors have loss of expression of one or more Mismatch Repair proteins. MSI tumors have better survival rate than microsatellite stable (MSS) tumors, poor response to 5FU-based adjuvant chemotherapy and relatively successful immunotherapy in metastatic MSI tumors. Immunohistochemistry recognizes altered gene by recognizing loss of its protein product. Based on the presence or absence of Mismatch repair proteins, groups are classified into Mismatch repair proficient (MMR-p) and Mismatch repair deficient (MMR-d). Aim: To investigate the immunohistochemical profile of Mismatch repair proteins namely: hMLH1, hMSH2, hMSH6, and hPMS2 in surgically resected colorectal cancer specimens. Materials and Method: A total of 76 cases were selected from the Histopathology Department of HTAA to determine MMR protein expression status. Cases were either MMR-p or MMR-d. Results: Of the specimens which were properly immunostained, seventeen out of seventy-six cases $(22.37 \%)$ showed loss of one or more MMR proteins expression and thus were MMR-d. MLH1, MSH2, MSH6 and PMS2 protein expression was detected as 85.53\% (65/76), 81.6\% (62/76), 88.16\% (67/76), and 76.32\% (58/76), respectively. Conclusion: Mismatch repair proteins profile should be done using immunohistochemistry in local laboratories on these selected cases before referring for the expensive molecular test. 